\title{
Drag force on a particle straddling a fluid interface: influence of interfacial deformations
}

\author{
Loudet J.-C. ${ }^{\dagger \ddagger, 1}$, Qiu M. ${ }^{\ddagger}$, Hemauer J. ${ }^{\ddagger}$, and Feng J.J. ${ }^{\ddagger}$ \\ †University of Bordeaux, CNRS, Centre de Recherche Paul Pascal (UMR 5031), \\ F-33600 Pessac, France \\ ${ }^{\ddagger}$ University of British Columbia, Department of Mathematics, Vancouver, BC, V6T 1Z2, \\ Canada
}

\begin{abstract}
We numerically investigate the influence of interfacial deformations on the drag force exerted on a particle straddling a fluid interface. We perform finite element simulations of the two-phase flow system in a bounded two-dimensional geometry. The fluid interface is modeled with a phase-field method which is coupled to the Navier-Stokes equations to solve for the flow dynamics. The interfacial deformations are caused by the buoyant weight of the particle, which results in curved menisci. We compute drag coefficients as a function of the three-phase contact angle, the viscosity ratio of the two fluids, and the particle density. Our results show that, for some parameter values, large drag forces are not necessarily correlated with large interfacial distortions and that a lower drag may actually be achieved with non-flat interfaces rather than with unperturbed ones.
\end{abstract}

Keywords: Two-phase flows, particle/fluid flow, fluid interface, phase-field simulations, interfacial deformations, drag force.

\footnotetext{
${ }^{1}$ jean-christophe.loudet@u-bordeaux.fr or jcloudet@math.ubc.ca
} 


\section{Introduction}

The problem of particles straddling an interface between two immiscible fluids (e.g. oil and water or air and water) has been extensively addressed in the literature for many decades. It is well-established that the presence of a fluid interface alters drastically the transport properties of adsorbed particles (e.g. macromolecular proteins, small Brownian aggregates, or macroscopic objects) and their interactions compared to the bulk situation. For instance, in addition to bulk interactions due to van der Waals and electrostatic forces, floating particles may also experience capillary interactions if they cause sufficient perturbations in the interfacial shape $[1,2]$. Such interactions originate from the overlap of interfacial distortions and feature energies well above the thermal energy that may lead to the clustering of a great variety of particles (from $10 \mathrm{~nm}$ to $1 \mathrm{~cm}$ ) forming a wide range of self-assembled structures $[1,3,4]$. Other experiments revealed that the diffusivity of Brownian interfacial particles may be at odds with the well-known bulk Stokes-Einstein law $[5]$.

Early investigations of interfacial particle hydrodynamics dealt with translational motions both across and along fluid phase boundaries combined with rotations about axes lying in the interfacial plane [6]. One of the simplest situations deals with the drag force $\mathbf{F}_{D}$ exerted on an isolated rigid sphere translating along a fluid interface at low Reynolds number. In this case, $\mathbf{F}_{D}$ has been written as [7]:

$$
\mathbf{F}_{D}=-6 \pi \mu R f_{d} \mathbf{U}
$$

where $R$ is the sphere radius, $\mu$ denotes a characteristic dynamic viscosity, $\mathbf{U}$ is the particle velocity relative to the fluids, and $f_{d}$ is a corrective dimensionless drag coefficient accounting for the presence of the interface. The well-known bulk Stokes drag is obviously recovered for $f_{d}=1$. However, this is seldom the case at the interface where $f_{d}$ can be either smaller or greater than 1. Several experimental and theoretical studies have shown that $f_{d}$ depends not only on the three-phase contact angle $\alpha$ and the viscosity ratio of the two fluids $[7,8,9,10,11,12,13]$, but also on the deformation of the interface which in turn is a function of the fluids density ratio, surface tension, size and density of the adsorbed 
particle [14]. The presence of adsorbed surfactants brings in an additional contribution to the drag, with $f_{d}$ up to $\simeq 3$, due to the viscous nature of the interface with nonvanishing shear and dilatational viscosities $[8,9,15,16,17]$. More recent investigations focused on the influence of contact line fluctuations on the drag force $[18,19]$, whereas both the rotational and translational dynamics of a colloid moving at a flat air-liquid interface in a thin film were addressed in [20].

However, among the various parameters affecting the value of $f_{d}$, the influence of interfacial deformations has been particularly overlooked so far. On the experimental side, Petkov et al. [14] were the first to address this issue and reported substantial $f_{d}$ values $\left(f_{d} \simeq 1.75\right)$ due to a deformed meniscus forming around heavy copper spheres translating at the air-water interface. The meniscus dimple was caused by the particle buoyant weight in this case and the authors attributed the enhanced drag coefficient to the motion of the curved meniscus around the particle together with the particle itself. So far, we are unaware of any other study similar to that of [14] at liquid-gas interfaces. However, a few works dedicated to pairwise interactions of interfacial particles at liquid-liquid interfaces mentioned smaller $f_{d}$ values, around 1.2, presumably for the same reasons $[21,22,23]$. On the theoretical side, only the recent work of Dörr \& Hardt [24] considered explicitly interfacial deformations around spherical particles translating along an interface between fluids of very different viscosities. The driven spheres act as capillary dipoles but the effect on the drag force was not reported. Furthermore, we are not aware of any numerical simulations that take explicitly into account interfacial perturbations. Does a curved meniscus always increase the drag, as is commonly believed? A detailed investigation on that subject is currently missing and will be the main topic of the present work. Since drag forces are ubiquitous in any fluid media, the problem is also relevant to the self-assembling properties of a great variety of adsorbed species (e.g. nano- or micro-particles, proteins, molecular aggregates) confined in fluid interfaces, thin liquid films, and even membranes of lipid vesicles or living cells [4].

In the following, we wish to shed some light on the influence of interfacial deformations on the drag force exerted on particles translating along a fluid interface. We tackle this 
task through two-dimensional (2D) numerical simulations able to describe the dynamics of two-phase flow systems in the presence of a fluid interface and a three-phase contact line. Our primary objective is to qualitatively capture the main physical trends upon browsing the parameter space, which justifies a simple 2D approach to start with. To the best of our knowledge, we do not know of any other simulations or theoretical works addressing similar issues.

The outline of the paper is as follows. In section 2, we describe the geometry of the problem prior to summarizing the governing equations in section 3. We specify the relevant dimensionless parameters along with some details relative to the computations of drag forces and particle densities. General considerations on the numerical method we employed follow in section 4 . Validation steps are then presented in section 5 before describing and discussing the results in section 6. In particular, we will show that large drag forces are not necessarily correlated with large interfacial deformations. Concluding remarks close the paper in section 7.

\section{Geometry}

The detailed geometry of our 2D problem is specified in Fig. 1. An infinitely long circular cylinder of radius $R$ and density $\rho_{p}$ is trapped at the interface between two Newtonian fluids of different densities $\left(\rho_{1}, \rho_{2}\right.$ with $\left.\rho_{2}>\rho_{1}\right)$ and dynamic viscosities $\left(\mu_{1}, \mu_{2}\right)$. The whole system is confined in a box of length $L$ and height $H$. In practice, the cylinder moves horizontally across the interface with a constant velocity $-U_{0} \hat{\mathbf{x}}$ under the action of an external force. However, in our simulations, we use a reference frame attached to the cylinder so that far from it, both liquids flow with constant velocity $U_{0}$ in the direction of the $x$-axis. In this case, the upper and lower bounding plane walls should also move with the same velocity $U_{0}$ from left to right in their own planes. Note that the cylinder's center of mass will not be necessarily located midway across the fluid interface. Actually, its position will be shifted either up or down in order to simulate different cylinder densities, as we will explain further below. In most cases, the fluid interface will be deformed around the cylinder, featuring either an upward or a downward meniscus depending on 
operating conditions. The interfacial tension between the two fluids is denoted as $\sigma$. The fluid interface is kept horizontal at the inlet and the outlet in all cases, whereas the contact angle, $\alpha$, at the cylinder surface (cf. Fig. 1) is a parameter of the simulation which will take on different values. All the physical and geometrical parameters used in the simulations are reported in table 1.

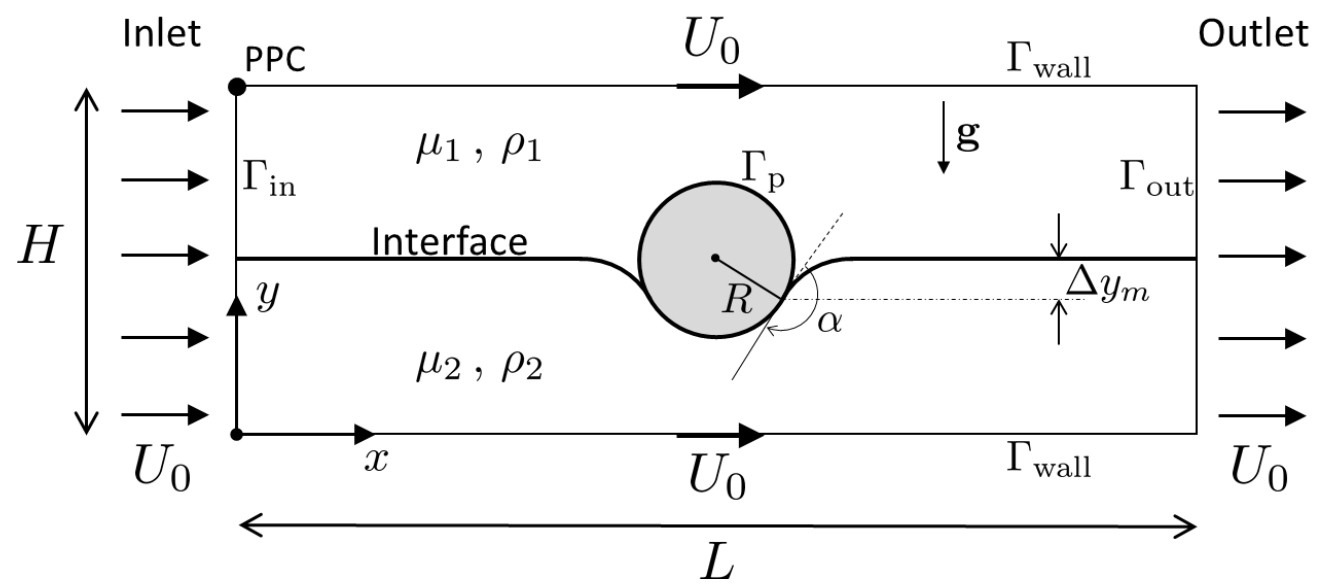

Figure 1: Geometry of the problem for the computation of two-dimensional two-phase flow drag on a circular cylinder confined at a fluid interface between two plane walls. PPC: Pressure Point Constraint, $\alpha$ : contact angle, $\Delta y_{m}$ : Meniscus elevation. See text and table 1 for other symbol definitions.

\section{Governing equations}

\subsection{Two-phase flow modeling}

In order to determine the drag force exerted on the cylinder at the fluid interface, we need a model able to describe the interfacial dynamics accurately. Among the numerous multiphase flow approaches available in the literature [25], we opted for a diffuse interface method, based on the concept of "phase-field" (PF), to account for the fluid interface motion, combined with the Navier-Stokes equations to describe the flow. The choice of the PF model was primarily motivated by the fact it provides an elegant, coarsegrained physical description of interfaces where short-range intermolecular interactions, 
Table 1: Definitions and values of the parameters used in the simulations.

\begin{tabular}{l|ccc}
\hline \hline Parameter & Symbol & Value & Unit \\
\hline \hline Cylinder radius & $R$ & 1 & $\mathrm{~mm}$ \\
Box length & $\mathrm{L}$ & $30 R$ & $\mathrm{~mm}$ \\
Box height & $\mathrm{H}$ & $20 R$ & $\mathrm{~mm}$ \\
Inlet/outlet velocity & $U_{0}$ & 20 & $\mu{\mathrm{m} . \mathrm{s}^{-1}}$ \\
Upper fluid dynamic viscosity & $\mu_{1}$ & $0.001-0.1$ & Pa.s \\
Lower fluid dynamic viscosity & $\mu_{2}$ & 0.1 & Pa.s \\
Upper fluid density & $\rho_{1}$ & $800-10^{3}$ & $\mathrm{~kg} \cdot \mathrm{m}^{-3}$ \\
Lower fluid density & $\rho_{2}$ & $10^{3}$ & $\mathrm{~kg} . \mathrm{m}^{-3}$ \\
Cylinder density & $\rho_{p}$ & $600-1200$ & $\mathrm{~kg} . \mathrm{m}^{-3}$ \\
Interfacial tension between fluids 1 and 2 & $\sigma$ & 0.01 & $\mathrm{~N} . \mathrm{m}^{-1}$ \\
Contact angle & $\alpha$ & $40^{\circ}-140^{\circ}$ & $\mathrm{deg}$ \\
\hline \hline
\end{tabular}

such as van der Waals forces, are implicitly taken into account. An explicit tracking of the fluid interface is unnecessary and the interface normal and curvature are not required to accurately determine surface tension forces. Moreover, the PF approach is naturally able to simulate contact line motion without any singularity or ad hoc slip models [25]. The details of the coupled phase-field/Navier-Stokes equations have been described extensively by a number of authors [25, 26, 27, 28]. Consequently, in the following, we will only highlight the main ideas and list the governing equations.

The two fluid components are considered immiscible, but in the PF formulation, they are allowed to mix within a thin interfacial region. A PF variable, or scaled 'concentration', $\phi$ is introduced such that in the two-fluid bulks $\phi= \pm 1$ and the fluid-fluid interface is defined by $\phi=0$. The free energy associated with the mixing process may be written

$$
f_{\text {mix }}=\frac{\lambda}{2}|\nabla \phi|^{2}+\frac{\lambda}{4 \epsilon^{2}}\left(\phi^{2}-1\right)^{2}
$$

where $\lambda$ is the mixing energy density with a dimension of force, and $\epsilon$ is a capillary width 
representative of the diffuse interface thickness. The equilibrium fluid-fluid interfacial tension is defined by the ratio $\lambda / \epsilon$ and given by $[26,27]$

$$
\sigma=\frac{2 \sqrt{2}}{3} \frac{\lambda}{\epsilon}
$$

The evolution of $\phi$, and therefore of the fluid interface, is governed by the Cahn-Hilliard $(\mathrm{CH})$ equation $[26,27]$

$$
\frac{\partial \phi}{\partial t}+\mathbf{v} \cdot \nabla \phi=\nabla \cdot(\gamma \nabla G)
$$

where $G=\lambda\left[-\nabla^{2} \phi+\left(\phi^{2}-1\right) / \epsilon^{2}\right]$ is the chemical potential, $\gamma$ the mobility parameter (assumed to be constant), and $\mathbf{v}$ the fluid velocity. The balance equations of mass and momentum must be added to the above relation to describe the fluid dynamics. The fluids being incompressible, the velocity and pressure fields satisfy the Navier-Stokes equations

$$
\begin{aligned}
\boldsymbol{\nabla} \cdot \mathbf{v} & =0 \\
\rho(\phi)\left(\frac{\partial \mathbf{v}}{\partial t}+\mathbf{v} \cdot \boldsymbol{\nabla} \mathbf{v}\right) & =-\boldsymbol{\nabla} p+\boldsymbol{\nabla} \cdot \underline{\boldsymbol{\sigma}}^{\boldsymbol{v}}+\rho(\phi) \mathbf{g}+G \boldsymbol{\nabla} \phi
\end{aligned}
$$

where the last body force term in the momentum relation $(G \boldsymbol{\nabla} \phi)$ is the diffuse-interface equivalent of the interfacial tension $[26,29] . \underline{\boldsymbol{\sigma}}^{\boldsymbol{v}}=\mu(\phi)\left[\boldsymbol{\nabla} \mathbf{v}+(\boldsymbol{\nabla} \mathbf{v})^{T}\right]$ is the viscous stress tensor, $\mathbf{g}$ is the gravitational acceleration, and $\rho(\phi)$ (resp. $\mu(\phi)$ ) is the density (resp. the viscosity) of the two-phase system given by: $\rho(\phi)=\frac{1+\phi}{2} \rho_{1}+\frac{1-\phi}{2} \rho_{2}$, and $\mu(\phi)=\frac{1+\phi}{2} \mu_{1}+\frac{1-\phi}{2} \mu_{2}$.

The coupled equations (4-6) must be supplemented by initial and boundary conditions. With the help of Fig. 1, the boundary conditions for the velocity are:

$$
\begin{aligned}
& \mathbf{v}=U_{0} \hat{\mathbf{x}}, \text { on } \Gamma_{\text {wall }} \\
& \mathbf{v}=\mathbf{0}, \text { on the cylinder }\left(\Gamma_{p}\right) \\
& \mathbf{v}=U_{0} \hat{\mathbf{x}}, \text { on } \Gamma_{\text {in }} \text { and } \Gamma_{\text {out }} .
\end{aligned}
$$

The pressure level was set to zero at the top left corner of the box [pressure point constraint (ppc) in Fig. 1]. As for the $\mathrm{CH}$ variables, we impose the following boundary 
conditions for the chemical potential $(G)$ and $\phi$

$$
\begin{aligned}
& \boldsymbol{\nabla} \cdot \hat{\mathbf{n}}=0, \text { on all boundaries } \\
& \boldsymbol{\nabla} \phi \cdot \hat{\mathbf{n}}=|\boldsymbol{\nabla} \phi| \cos \alpha, \text { at the contact line } \\
& \boldsymbol{\nabla} \phi \cdot \hat{\mathbf{n}}=0, \text { on } \Gamma_{\text {in }} \text { and } \Gamma_{\text {out }},
\end{aligned}
$$

where $\hat{\mathbf{n}}$ is the outward unit normal vector to the considered boundary. Eq. (10) is a zero-flux natural boundary condition resulting from the variational formulation of the $\mathrm{CH}$ equation. Eq. (11) is a geometric condition enforcing a constant contact angle $\alpha$ at the three-phase contact line on the cylinder. This condition is set in the Comsol phasefield module and allows the determination of the contact line position. The latter is automatically updated according to the motion of the fluid interface. Also, we assume that equilibrium is reached at any time so that contact angle hysteresis is discarded. Outside of the fluid interface, Eq. (11) reduces to a homogeneous Neumann condition, while Eq. (12) simply reflects the fact that we impose $\alpha=90^{\circ}$ on $\Gamma_{\text {in }}$ and $\Gamma_{\text {out }}$.

Finally, as initial conditions, we set up a zero pressure and fluid velocity everywhere in the domain. At time $t=0$, the fluid interface is flat and the particle center of mass lies in the center of the box, if not otherwise stated.

\subsection{Dimensionless groups}

If we consider the parameters listed in table 1 , together with those specific to the $\mathrm{CH}$ model, namely, the capillary width $\epsilon$ and the interfacial mobility $\gamma$, a total of seven 
dimensionless numbers may be constructed for our problem:

$$
\begin{aligned}
B o & =\frac{\Delta \rho g R^{2}}{\sigma}, \text { where } \Delta \rho=\rho_{2}-\rho_{1} \quad \text { (Bond number) } \\
C a & =\frac{\mu_{2} U_{0}}{\sigma}, \quad \text { (capillary number) } \\
R e & =\frac{\rho_{2} U_{0} R}{\mu_{2}}, \quad \text { (Reynolds number) } \\
C n & =\frac{\epsilon}{R}, \quad(\text { Cahn number) } \\
S & =\frac{\sqrt{\gamma \mu_{e}}}{R}, \text { where } \mu_{e}=\sqrt{\mu_{1} \mu_{2}} \quad \text { (mobility number) } \\
\mu^{*} & =\frac{\mu_{1}}{\mu_{2}}, \quad(\text { viscosity ratio) } \\
\rho^{*} & =\frac{\rho_{1}}{\rho_{2}}, \quad \text { (density ratio) } .
\end{aligned}
$$

The Bond number, $B o$, compares the relative importance of surface tension and gravity forces. It may also be written as: $B o=\left(R / L_{c}\right)^{2}$, where $L_{c}=\sqrt{\sigma /(g \Delta \rho)}$ is the capillary length. The latter gives the typical length scale over which interfacial deformations occur due to the competition between surface tension and gravity. In our work, $B o$ will be smaller than unity $(B o \simeq 0.2)$, meaning that capillarity will be more important than gravity. The capillary number will be very small in this study, $C a \sim 10^{-4}-10^{-3}$, implying that the shape of the fluid interface should not be altered by the flow around the cylinder. This is the simplest situation to consider as a first step and, given the already complicated nature of the problem, we will not explore here the effect of finite $C a$, which can be examined in a future effort. Moreover, since the cylinder is kept fixed and does not rotate, and that all our drag simulations involve steady-state flows, there is no motion of the contact line relatively to the cylinder surface.

Next, fluid inertia is not important here since the Reynolds number is well below unity $\left(R e \sim 10^{-4}-10^{-3}\right)$, i.e. we are near the Stokes flow limit. The two following dimensionless groups, $\mathrm{Cn}$ and $\mathrm{S}$, are specific to the $\mathrm{CH}$ model. The former represents the interfacial thickness while the latter reflects the so-called CH-diffusion across the fluid interface [30]. Their values cannot be easily assigned for real materials but are of paramount importance to ensure convergence and accuracy of the computed solutions. We used the guidelines proposed in $[29,30]$ to ensure that the fluid interface was appropriately resolved, which is 
a crucial issue in the PF method. More details on this point will be provided in the next section. Finally, we varied the viscosity ratio in the range $0.01-1$ while the density ratio was kept close to unity $(0.8-1)$.

\subsection{Drag force and particle density calculations}

In our 2D geometry (Fig. 1), the drag force (per unit length), $\tilde{F}_{D}$, acting on the particle in the $x$-direction, can be computed by integrating the total stress exerted on the cylinder contour, $\Gamma_{p}$,

$$
\tilde{F}_{D}=\hat{\mathbf{x}} \cdot \oint_{\Gamma_{p}}\left(-p \underline{\mathbf{I}}+\underline{\boldsymbol{\sigma}}^{v}\right) \cdot \hat{\mathbf{n}} d s
$$

where $\hat{\mathbf{x}}$ is the unit vector in the $x$-direction, $\hat{\mathbf{n}}$ the outward unit normal vector on the line element $d s$, and $\underline{\boldsymbol{\sigma}}^{v}$ the viscous stress tensor defined previously [Eq. (6)]. Since we only consider the case of a nonviscous fluid interface, there is no additional contribution coming from the fluid interface to the drag force. The influence of interfacial viscosity on $\tilde{F}_{D}$ was considered by, e.g. Danov et al. $[8,9]$.

In our simulations, the interfacial deformations are caused by the particle's buoyant weight which depends on its density. At steady state, the $y$-component of the force balance on the particle reads:

$$
\tilde{M} g=\tilde{F}_{v}+\tilde{F}_{c}=\hat{\mathbf{y}} \cdot \oint_{\Gamma_{p}}\left(-p \underline{\mathbf{I}}+\underline{\boldsymbol{\sigma}}^{v}\right) \cdot \hat{\mathbf{n}} d s+\sigma \hat{\mathbf{y}} \cdot \oint_{\Gamma_{p}} \underline{\boldsymbol{\sigma}}^{c} \cdot \hat{\mathbf{n}} d s
$$

where $\tilde{M}$ is the mass (per unit length) of the cylinder and $\hat{\mathbf{y}}$ is the unit vector in the $y$-direction. $\underline{\boldsymbol{\sigma}}^{c}$ is the capillary stress tensor whose expression can be derived using a variational procedure, as shown in [27], yielding

$$
\underline{\boldsymbol{\sigma}}^{c}=f_{\mathrm{mix}} \underline{\mathbf{I}}-\lambda \boldsymbol{\nabla} \phi \boldsymbol{\nabla} \phi
$$

where $f_{\text {mix }}$ is the mixing free energy density defined in Eq. (2). Note that taking the divergence of Eq. (22) leads exactly to the body force term $(G \nabla \phi)$ in Eq. (6) for the surface tension. The first term on the right hand side (rhs) of Eq. (21) accounts for the viscous force (per unit length), $\tilde{F}_{v}$, exerted on the particle due to stresses generated 
by fluid motion. In the absence of flow, $\tilde{F}_{v}$ simply reduces to the buoyancy force. The second term on the rhs of Eq. (21) corresponds to the capillary force (per unit length), $\tilde{F}_{c}$, exerted on the cylinder because of interfacial deformations. Note that $\tilde{F}_{c}$ becomes non-zero as soon as the interface deforms to satisfy the prescribed contact angle for a given position of the cylinder across the interface. Since $\tilde{F}_{v}$ and $\tilde{F}_{c}$ can be readily computed in the simulations, the particle weight (or density) follows directly from Eq. (21).

In this work, we would like to stress that we choose to explore the effect of different particle densities not by tuning the value itself, but by changing the vertical position of the particle across the interface. For a given contact angle, we compute the steady state solution while holding the cylinder fixed in place and compute its density as explained above. We will demonstrate in Sec. 5.2 that the resulting interfacial profile is identical to that one would obtain by solving explicitly the vertical force balance [Eq. (21)] for a freely moving cylinder of the same density. In the latter case, the cylinder would be allowed to translate vertically across the interface until equilibrium is reached, and a costly moving mesh feature would be required to resolve this motion in situ. Our strategy enables us to bypass this complication, thereby making the computations faster.

Finally, it will be shown in Sec. 6.3 that the explored range of particle displacements across the interface does not incur any change in confinement effect.

\section{Numerical method}

Eqs. (4)-(6), and the imposed boundary conditions [Eqs. (7)-(12)], were solved numerically using the finite element computational software COMSOL Multiphysics ${ }^{\circledR}$ [31] with the coupled two-phase laminar flow/phase-field (Cahn-Hilliard) modules. To guarantee accuracy and stability, we discretized the fluid flow with quadratic elements for the velocity field and linear elements for the pressure field; quadratic elements were employed to discretize the phase-field variable. We used a fixed nonuniform triangular mesh fitted with subdomains to appropriately resolve the fluid interface as it moved within the domain to satisfy the imposed contact angle on the cylinder surface. The subdomains were uniformly meshed with a mesh size adjusted so that the interfacial thickness, which is on 
the order of $4 \epsilon$, contained at least $8-10$ elements. According to the criterion defined in $[29,30]$, the latter condition ensures a sufficient resolution of the fluid interface, which is of great importance within the PF framework. The dimensions of the subdomains were optimized in each case, depending on the contact angle value and the vertical position of the cylinder. Sizeable interfacial deformations, which mostly occurred for small $\left(\alpha<50^{\circ}\right)$ and large $\left(\alpha>130^{\circ}\right)$ contact angles, required the largest subdomains, as expected. Outside of these domains, the mesh size was much coarser since the fluid interface never wandered in these areas. The value of the mobility number $S$ was adjusted so that the inequality $C n<4 S$ was always satisfied in all our simulations. The latter condition was recommended as a guideline in [30] for producing convergent results with moving contact-

lines. With a Cahn number $C n=0.04$ (resp. $C n=0.02$ ), a typical mesh consisted of $\simeq 140000$ (resp. 450000 ) elements with about 1.5 million (resp. 4.8 million) degrees of freedom. We advanced the system to steady state using an implicit time-stepping scheme. At every time step, we used Newton iterations to solve the nonlinear system, and chose either the 'MUMPS' or 'PARDISO' solver of COMSOL to solve linear systems. Steady state was reached, in most cases, after $\sim 50 \mathrm{~s}$ (physical time) and about $1 \mathrm{~h}(\mathrm{Cn}=0.04)$ to $4 \mathrm{~h}(C n=0.02)$ of computing time on a workstation equipped with an Intel i7 8-core processor with 64 GB of RAM.

\section{Validations}

\subsection{Drag force}

Prior to dealing with non-flat interfaces, we first present a validation of our numerical setup for computing the drag force on the cylinder in a confined two-phase flow case with a flat interface. Since the simulation box has a finite size, confinement effects are unavoidable, and it is well-known that the presence of boundaries strongly influence drag values [32]. Nevertheless, the confined case is still very interesting to consider because, one the one hand, experimental systems are always confined, and on the other hand, the so-called Stokes' paradox [33] disappears in a bounded geometry, allowing 2D Stokes drag 
Table 2: Comparison between our numerical results and those of the literature for the dimensionless drag force $\bar{F}_{D}$. Definition of symbols: $H / L$ : Box aspect ratio; spf: single-phase flow; tpf: two-phase flow. Rel. err.: Relative error between Present Study - spf and Present Study - tpf. Cn: Cahn number [cf. Eq. (16)]. For the Present Study - tpf case, the two phases have the same viscosity and density than in the Present Study - spf situation. For illustrative purposes, only the results for two different values of $H / L$ are displayed below, but many other degrees of confinement were tested and show an equally good agreement between the literature data and our simulations. Parameters: $\mu=0.1$ Pa.s, $\rho=10^{3} \mathrm{~kg} \cdot \mathrm{m}^{-3}, U_{0}=20 \mu \mathrm{m} . \mathrm{s}^{-1}$, $R e=2 \times 10^{-4}$.

\begin{tabular}{|c|c|c|c|c|c|}
\hline \multirow{2}{*}{$H / L$} & \multicolumn{5}{|c|}{$\bar{F}_{D}=\tilde{F}_{D} / \mu U_{0}$} \\
\hline & Ref. [35] & Ref. [34] & Present Study - spf & Present Study - tpf & Rel. err. (\%) \\
\hline \multirow{3}{*}{$20 R / 30 R$} & \multirow{3}{*}{9.1990} & \multirow{3}{*}{8.9506} & \multirow{3}{*}{8.9440} & $8.9650(C n=0.04)$ & 0.26 \\
\hline & & & & $8.9535(C n=0.02)$ & 0.13 \\
\hline & & & & $8.9400(C n=0.01)$ & 0.02 \\
\hline \multirow{3}{*}{$10 R / 20 R$} & \multirow{3}{*}{16.7335} & \multirow{3}{*}{16.5326} & \multirow{3}{*}{16.5160} & $16.5555(C n=0.04)$ & 0.25 \\
\hline & & & & $16.5155(C n=0.02)$ & 0.12 \\
\hline & & & & $16.5080(C n=0.01)$ & 0.05 \\
\hline
\end{tabular}

analytical solutions to be found [34]. However, since we were unable to find any reference data for two-phase flows in a confined geometry, we used as references the analytical result of Faxén [34] and the numerical data of Ben Richou et al. [35] valid for single-phase flows and very low Re. To compare with such data, we computed the bulk flow around a cylinder, first using a single-phase formalism, and then employing our more general two-phase formalism. In the latter case, we matched the densities and viscosities of the two phases so that the fluid interface plays no role. In these tests, the cylinder lies in the middle of the box with the fluid interface (when present) halving the domain. In two-phase flows, a $90^{\circ}$-contact angle was imposed on all solid boundaries. We computed the dimensionless drag force $\bar{F}_{D}=\tilde{F}_{D} / \mu U_{0}$ for two box aspect ratios $H / L$. The values are summarized in table 2 , along with the other relevant parameters. 


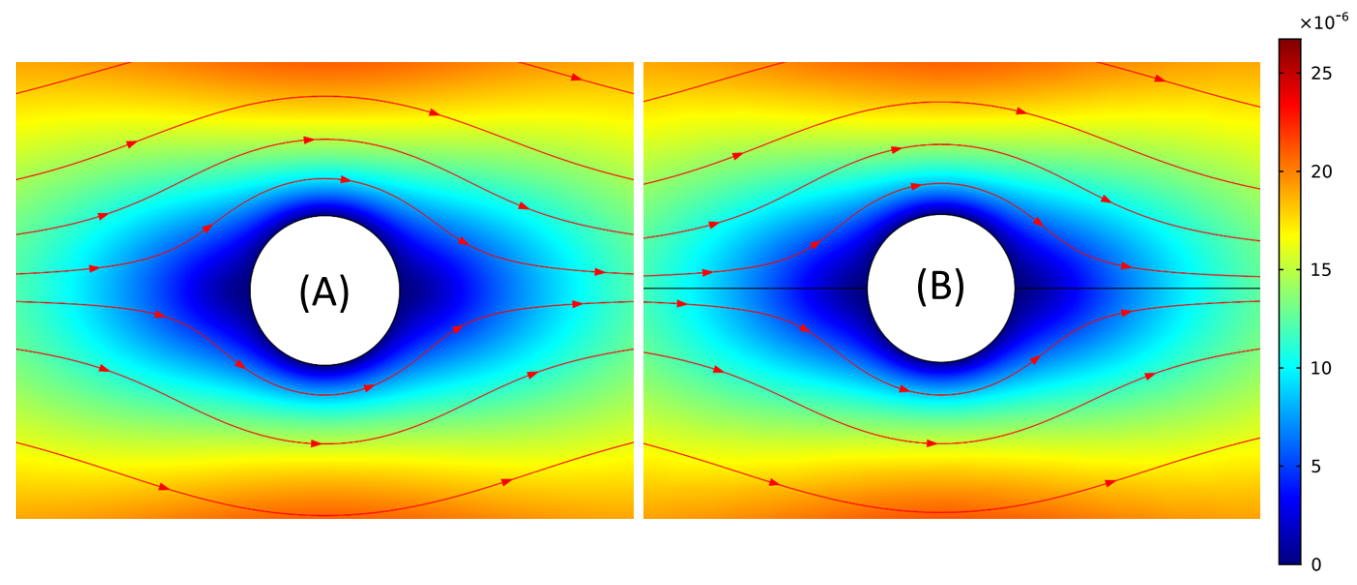

Figure 2: Velocity field and the associated streamlines (red arrow lines) obtained for (A) a single-phase flow (spf) (reference state), and (B) a two-phase flow (tpf) with the cylinder held fixed in the middle of the box. The fluid interface is represented as a solid black line $(\phi=0)$ and the color bar indicates the velocity amplitude in $\mathrm{m} / \mathrm{s}$. Simulation parameters: $\alpha=90^{\circ}$ (tpf), $\mu^{*}=1$ (tpf), $\rho^{*}=1$ (tpf), $C n=0.01$ (tpf), $H / L=20 R / 30 R$.

As can be seen from table 2 , our single-phase flow values for $\bar{F}_{D}$ reproduce well the literature data, especially those due to Faxén [34]. The same comment goes for the two-phase flow situation with identical phases. The relative error on $\bar{F}_{D}$ is on the order of $0.25 \%$ or less depending on the Cahn number. As $C n$ decreases, the two-phase flow value of $\bar{F}_{D}$ becomes extremely close to its single-phase flow counterpart, meaning that we approach the so-called sharp interface limit $(\epsilon \rightarrow 0)[29,30]$. In this limit, the $\mathrm{CH}$ diffusion is minute, the fluid interface is at equilibrium and does not perturb the streamlines, which should be identical (with $\alpha=90^{\circ}$ ) to those of the single-phase flow case, as is indeed revealed in Fig. 2. The overall good agreement therefore validates our numerical setup.

In the following, we will use the box size $H / L=20 R / 30 R$ to make sure that (i) the position of the confining walls is not too close to the cylinder, i.e. we are not dealing with a highly confined geometry, and (ii), the lateral extension of the fluid interface covers a few times the capillary length in most cases. Note that increasing the length of the simulation box is computationally costly since the fluid interface has to be finely resolved within the PF method. For $H=20 R$, we have checked that increasing $L$ further has 
no consequence on the value of $\bar{F}_{D}$ (the variation is less than $0.01 \%$ ), and therefore, we will keep $L=30 R$. Also, for most simulations, we have chosen either $C n=0.04$ or $C n=0.02$, which turned out to be good enough values realizing a tradeoff between precision and reasonable computing time. Lower values of $C n$ do increase the accuracy of $\bar{F}_{D}$ (cf. table 2), but at the expense of much longer computations. Finally, we have checked that the mass of both fluids is well-conserved in our simulations. The relative mass variation is typically less than $0.01 \%(0.005 \%)$ for $C n=0.04(C n=0.02)$.

\subsection{Meniscus profile and particle density}

In order to check the validity of the interfacial shapes, and the values of the particle density computed in the drag simulations, we performed a series of tests that we shall now outline.

Let us first consider a purely static situation where the velocity of the flow field at the inlet, outlet, and the bounding walls is set to zero. For a given set of physical parameters (particle density, contact angle, surface tension, and so forth), we then carry out a Comsol simulation which solves for the vertical force balance at the interface [cf. Eq. (21)]. In such a simulation, the particle is allowed to move in the $y$-direction depending on its weight. We used the built-in moving mesh module of Comsol based on an Arbitrary LagrangianEulerian (ALE) scheme to follow and resolve the particle motion until equilibrium is reached [36]. The resulting particle position $\left(y_{p}\right)$, meniscus elevation $\left(\left|\Delta y_{m}\right|\right.$, defined in Fig. 1), and interfacial profile $(y(x))$ are then compared with those obtained from an independent in-house code that solves the Young-Laplace equation in 2D and takes into account the aforementioned vertical force balance. Note that in the static case, analytical expressions can be derived for the capillary force and the buoyancy force acting on the floating cylinder [37]. We followed closely the procedure outlined by Pozrikidis and all the details can be found in [38].

A typical result of these static tests is displayed on Fig. 3 for a contact angle $\alpha=110^{\circ}$ and a particle density $\rho_{p}=982.2 \mathrm{~kg} / \mathrm{m}^{3}$. A very good agreement is achieved for the interfacial profiles as well as for other quantities such as those listed in table 3 (first two 


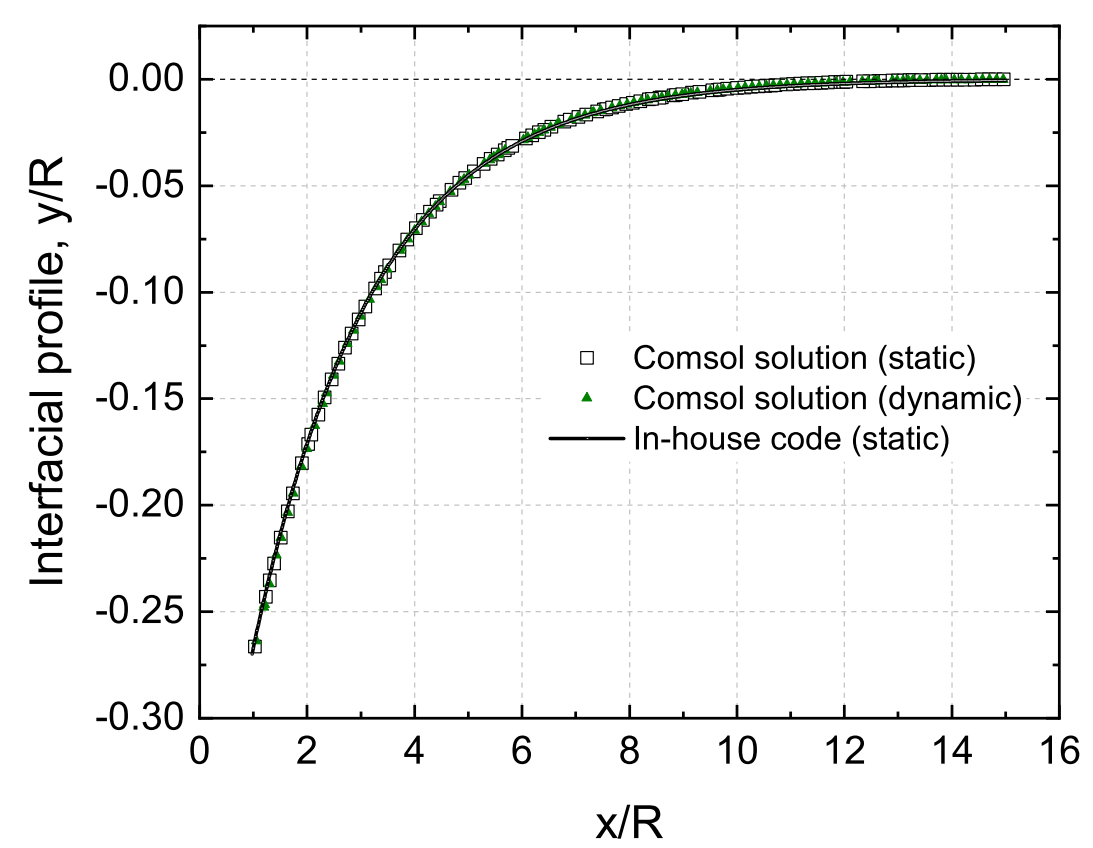

Figure 3: Meniscus profiles obtained for a cylinder attached at a fluid interface. Comparison of the Comsol simulation results (static and dynamic cases) with those computed from an independent in-house code (static case). See Sec. 5 for details. Parameters: $\alpha=110^{\circ}$, $\rho_{p}=982.2 \mathrm{~kg} / \mathrm{m}^{3}, \mu^{*}=1, \rho^{*}=0.8, C n=0.04$ (Comsol).

rows). Similar matches were realized upon testing a few other parameter values. The above results therefore validate our numerical approach in the static case.

Next, let us move on to the dynamic situation, i.e. the drag simulation setup of Fig. 1, where the interface is initially flat. We now fix the particle position $y_{p}$ at the value determined from the static case and choose the same contact angle on the particle surface. Note that the boundary conditions for $\phi$ at the inlet and outlet of the domain are the same in both the static and dynamic situations. The vertical position of the fluid interface hardly changes between the two configurations since it is required to be flat there (Sec. 3) with a minute $\mathrm{CH}$ diffusion at steady state. Then, because the capillary number is small in all our simulations (Sec. 3.2), we expect the dynamic interfacial profiles at steady state to differ very little from their static counterparts for the same set of physical 
Table 3: Comparison of the Comsol results with those of an in-house code computed for a cylinder floating at a fluid interface. All the variables are defined in the text (see also table 1 ). The physical parameters are the same as those listed in Fig. 3.

\begin{tabular}{l|ccccc} 
& $\Delta y_{m} / R$ & $y_{p} / R$ & $\tilde{F}_{c}\left[10^{-3} \mathrm{~N} / \mathrm{m}\right]$ & $\tilde{F}_{v}\left[10^{-2} \mathrm{~N} / \mathrm{m}\right]$ & $\rho_{p}\left[\mathrm{~kg} / \mathrm{m}^{3}\right]$ \\
\hline \hline In-house code & -0.269 & -0.0424 & 2.386 & 2.788 & 982.2 (input) \\
Comsol (static) & -0.273 & -0.0409 & 2.393 & 2.787 & 982.2 (input) \\
Comsol (dynamic) & -0.274 & -0.0421 & 2.400 & 2.787 & 982.186 \\
\hline \hline
\end{tabular}

parameters. Fig. 3 clearly demonstrates that this is indeed the case since all profiles are very well superimposed. This result allows us to accurately back out the particle density directly from the dynamic meniscus shape, as explained in Sec. 3.3. The very good match between static and dynamic data is quantitatively illustrated in the last row of table 3 .

\section{Results and discussion}

After the validation steps, we now address in detail the influence of interfacial deformations on the drag force exerted on the cylinder attached to the fluid interface. We will be focusing on the role of three parameters, namely (i) the contact angle, (ii) the viscosity ratio between the upper and lower fluids, and (iii) the particle density. We stress again that our 2D simulations are only likely to capture qualitative trends, but that this limitation does not prevent new physical insights from being unveiled, as we will show hereafter.

\subsection{Influence of the contact angle}

In the simulations to be described below, the cylinder is held fixed in the middle of the box $\left(y_{p} / R=0\right)$ and we vary $\alpha$ in the range $45^{\circ}-135^{\circ}$. We choose $\rho^{*}=0.8$, thereby giving $L_{c} / R \simeq 2.26$ and $B o \simeq 0.2$ using the values of table 1 . For each value of $\alpha$, we compute $\tilde{F}_{D}$ along with the meniscus elevations $\left|\Delta y_{m}\right|$. The numerical data are gathered in Figs. 4-5 for fluids with matched viscosities $\left(\mu^{*}=1\right)$. Note that the drag force has 
been normalized with respect to that calculated in the flat interface case, i.e. for $\alpha=90^{\circ}$. Hence, the effect of interfacial distortions can be readily assessed by a direct reading of the values $F_{D}^{*}=\tilde{F}_{D} / \tilde{F}_{D}\left(\alpha=90^{\circ}\right)$ on the $y$-axis.

In Fig. 4 , the curve $F_{D}^{*}=f(\alpha)$ has the shape of a symmetric concave parabola with a minimum obtained for $\alpha=90^{\circ}$ and $\left|\Delta y_{m}=0\right|$. Indeed, when $\alpha \neq 90^{\circ}$, the curved meniscus perturbs the flow streamlines with respect to the flat interface configuration, thereby increasing the friction. Furthermore, the highest drag values are correlated with the largest meniscus deformations, as expected. And since the two phases have equal viscosities, the perturbation is symmetric with respect to $\alpha=90^{\circ}$. Note that the small density difference between the two fluids $\left(\rho^{*}=0.8\right)$ does not perturb this symmetry, probably because inertia does not matter much here $(R e \ll 1)$.

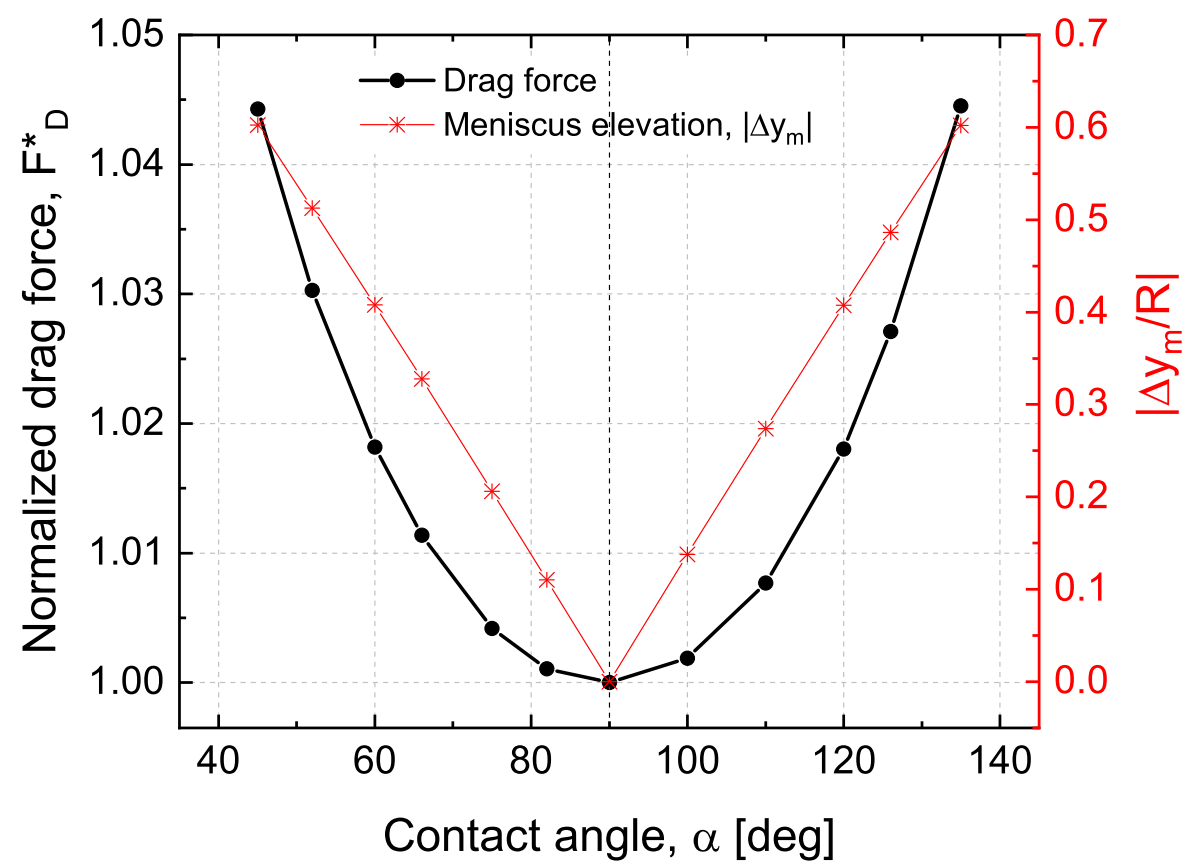

Figure 4: Computed normalized drag force $\left(F_{D}^{*}\right)$ and meniscus elevations $\left(\left|\Delta y_{m}\right|\right)$, as a function of the contact angle $(\alpha)$. Simulation parameters: $\mu^{*}=1, \rho^{*}=0.8, C n=0.04$.

Furthermore, we notice that $\left|\Delta y_{m}\right|$ varies linearly with $\alpha$ (Fig. 4). Such a linear dependence can be rationalized from the Young-Laplace equation and the associated boundary 
conditions governing the meniscus shape around the floating cylinder. The details of the derivation are presented in the appendix. With the notations of Fig. 9 (cf. appendix), it can be shown that

$$
\left|\Delta y_{m}\right|=2 L_{c} \sin \left(\frac{\alpha-\beta}{2}\right)
$$

where $\beta$ is the floating angle (Fig. 9). Within the explored range of $\alpha$ values, it turns out that the argument $(\alpha-\beta) / 2$ remains small and does not exceed $7.5^{\circ}$. Therefore, to first order, $\left|\Delta y_{m}\right| \approx L_{c}(\alpha-\beta)$, which explains the linearity of $\left|\Delta y_{m}\right|$ vs. $\alpha$ in Fig. 4.

Figs. 5a,b display typical velocity fields calculated for $\alpha=110^{\circ}$. When compared to Fig. 2b $\left(\alpha=90^{\circ}\right)$, the influence of the meniscus is clearly reflected by a deformed flow pattern. The PF map of Fig. 5c shows the interfacial deformations in the neighborhood of the cylinder. The corresponding mesh, superimposed on the PF map, is exhibited on Fig. 5d, which shows that the fluid interface is well-resolved within the subdomains, according to the criterion defined earlier (Sec. 4).

Overall, the data of Fig. 4 show that, for $\mu^{*}=1$, the effect of interfacial deformations on $F_{D}^{*}$ is rather moderate within the probed range of $\alpha$, yielding an excess drag, defined as $\left(F_{D}^{*}-1\right) \times 100$ (in \%), on the order of $5 \%$ compared to the case of a flat interface. Note that this order of magnitude compares well with that obtained by Danov et al. [9], who computed 3D excess drag coefficients for an infinite, flat, and nonviscous fluid interface with $\mu^{*}=1$. By shifting the particle up and down across the undistorted interface, which amounts to testing several contact angles, the authors report a similar concave shape for the curve $f_{d}=f(\alpha)$ with $f_{d}$ [cf. Eq. (1)] ranging up to 1.05. Hence, similarly to the curved meniscus, shifting the particle's vertical position breaks the symmetry of the flow pattern and results in an increased drag.

Experimentally, only a few studies considered the hydrodynamical behavior of particles trapped at fluid interfaces with matched viscosities (typically, oil-water systems). For instance, Vassileva et al. [21] and Boneva et al. [22, 23] tracked the trajectories of pairs of interacting sub-millimeter spheres and deduced an excess drag coefficient of around $10-$ $20 \%$, presumably ascribed to interfacial deformations. However, one should bear in mind that actual colloidal systems at fluid interfaces are far from "ideal" and feature a lot more 

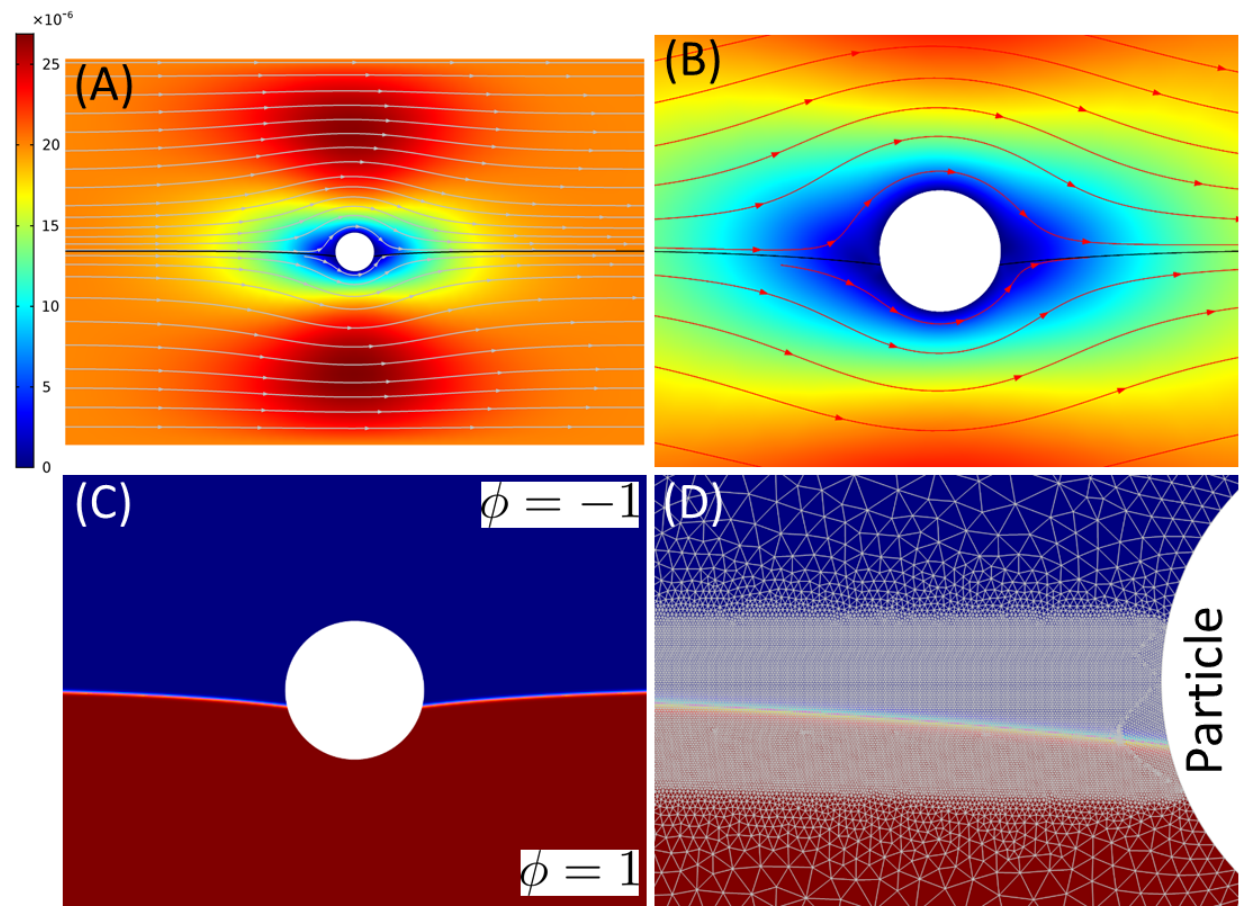

Figure 5: (A) Velocity field and the associated streamlines (gray arrow lines) computed for a contact angle $\alpha=110^{\circ}$ at steady state. The black solid line represents the fluid interface and the color bar indicates the velocity amplitude in $\mathrm{m} / \mathrm{s}$. (B) Zoomed-in view of (A) close to the particle with streamlines as red arrow lines. (C) Corresponding phase-field map with the fluid interface $(\phi=0)$ plotted as a solid magenta curve. The phase variable $\phi$ is specified for each fluid. (D) Zoomed-in view of a typical mesh superimposed to the phase-field map in the vicinity of the particle. The fluid interface is well-resolved since it always lies in a finely meshed region defined as a subdomain (cf. Sec. 4). Simulation parameters: $\alpha=110^{\circ}, \mu^{*}=1$, $\rho^{*}=0.8, C n=0.02$. 
complications. For instance, the particle surface roughness, chemical inhomogeneities, contact angle hysteresis or the ubiquitous presence of a variety of contaminants are only but a few of the phenomena which are likely to profoundly alter the response of the system and dash the hope of an easy comparison with idealized computations.

Hence, with the above proviso in mind, both simulations and experiments qualitatively agree on the fact that a deformed fluid interface, with matched viscosities, enhances only slightly the drag force on the particle.

\subsection{Influence of the viscosity ratio}

In this section, we examine how a viscosity difference between the two fluids affects the drag force. We will consider a two-order-of-magnitude change of $\mu^{*}$ in the range $0.01-1$, by altering the upper fluid viscosity $\left(\mu_{1}\right)$ whilst keeping that of the lower one $\left(\mu_{2}\right)$ constant (see table 1). The particle is held fixed at the center of the box $\left(y_{p}=0\right)$.

First, only a slight deviation of $\mu^{*}$ from unity is investigated as a function of the contact angle. With $\mu^{*}=0.75$, we see that the curve $F_{D}^{*}=f(\alpha)$ reported on Fig. 6 is no longer symmetric with respect to $\alpha=90^{\circ}$. Its minimum is shifted towards larger $\alpha$ and is now reached for $\alpha=100^{\circ}$. Furthermore, the highest drag force is obtained for $\alpha<90^{\circ}$, i.e. when the meniscus is convex and deformed upwards. Indeed, when $\alpha$ decreases, the size of the region underneath the meniscus grows as the meniscus becomes more and more distorted. This leads to an enhanced friction since the lower fluid is more viscous than the upper one. Equivalently, for $\mu^{*}>1$, the minimum drag would have been shifted towards $\alpha<90^{\circ}$ and the highest drag force would have occurred for $\alpha>90^{\circ}$. Furthermore, because of the small capillary number, note that the values of $\left|\Delta y_{m}\right|$ do not depend on $\mu^{*}$ and, consequently, they are the same here as those reported in Fig. 4.

Thus, for $\mu^{*} \neq 1$, our results show that one can encounter situations where the flat interface case $\left(\alpha=90^{\circ}\right)$ actually causes more friction than a slightly non-flat one. This finding departs from the common belief that interfacial disturbances yield necessarily an extra hydrodynamic resistance.

Note that, for $\mu^{*} \neq 1$, the drag force exerted on the particle should normally be 


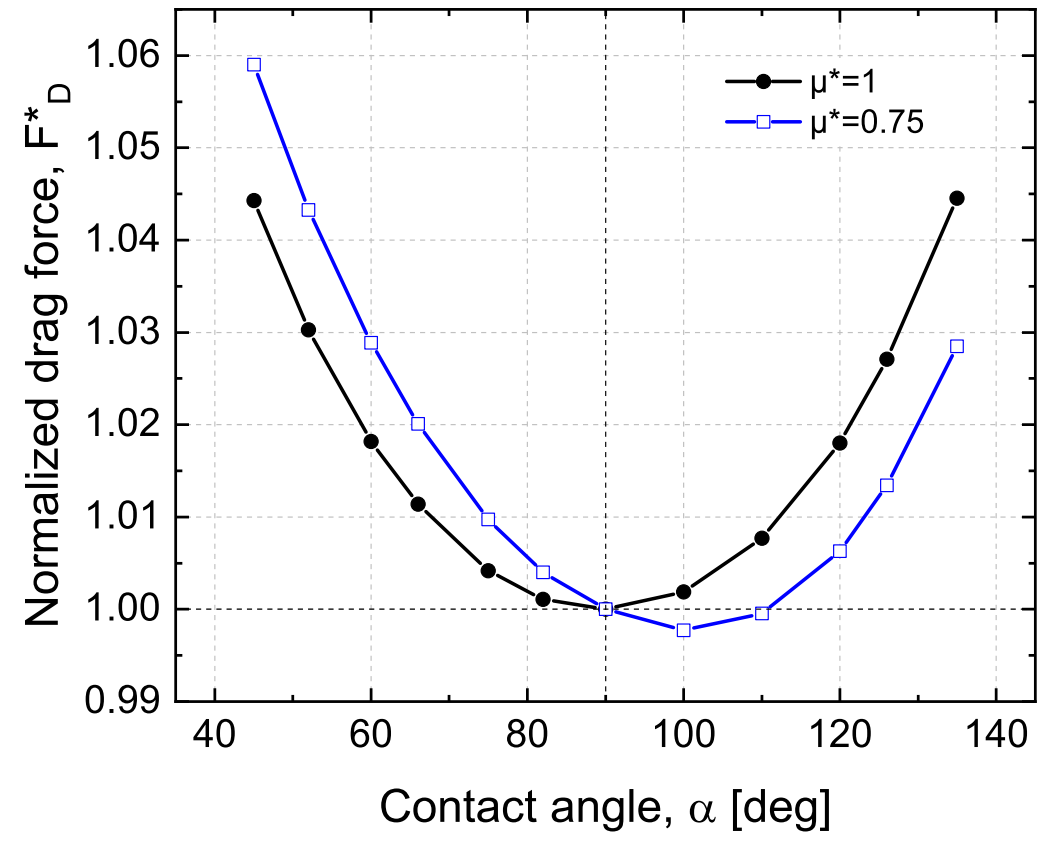

Figure 6: Computed normalized drag force $\left(F_{D}^{*}\right)$ as a function of the contact angle $(\alpha)$ for two viscosity ratios $\left(\mu^{*}\right)$. For $\mu^{*}=0.75$, the curve is no longer symmetric with respect to $\alpha=90^{\circ}$ and the minimum drag force is obtained for a slightly non-flat interface with $\alpha=100^{\circ}$. See text for further details. Simulation parameters: $\rho^{*}=0.8, C n=0.04$. 
accompanied by a non-zero viscous torque as well. The main reason for this is that, with fluids of different viscosity, the drag force's line of action does not generally run through the particle's center of mass, as first mentioned by Dörr \& Hardt [24]. Consequently, the particle is expected to rotate in this case. Actually, particle rotation is also expected to occur even for $\mu^{*}=1$ as long as there is an up-down asymmetry in the flow pattern. The latter may be caused either by interfacial distortions, as we saw in Sec. 6.1, or simply by shifting the particle position across an otherwise flat interface [9]. Anyway, as aforesaid (end of Sec. 3.2), we have ignored such a rotation in the present study, where the particle is kept fixed with respect to the passing flow. Allowing particle rotation implies that the contact line dynamics would have to be taken into account. Although the PF method is well-adapted to tackle such an issue $[25,30,39,40]$, we will leave it for a future investigation.

We also performed simulations with much larger viscosity mismatches, i.e. much smaller values of $\mu^{*}$. The graph of Fig. 7a represents dimensional drag force values plotted vs. $\mu^{*}$ for three different contact angles $\left(75^{\circ}, 90^{\circ}\right.$, and $\left.110^{\circ}\right)$. The data obtained for $\alpha=90^{\circ}$ serve as a reference with a flat fluid interface. Since inertia is negligible $(R e \ll 1)$, the drag force follows the expected linear dependence on $\mu^{*}$ for the three $\alpha$ 's. Next, we note that the data points for $\alpha=110^{\circ}$ mostly lie below those computed for $\alpha=90^{\circ}$, especially at low values of $\mu^{*}$. Upon re-plotting these data using normalized drag forces (Fig. $7 \mathrm{~b})$, we see more clearly that $F_{D}^{*}\left(\alpha=110^{\circ}\right)$ falls well below unity as $\mu^{*}$ decreases, while the opposite trend is observed for $F_{D}^{*}\left(\alpha=75^{\circ}\right)$. This result is a further evidence that the presence of interfacial deformations can actually yield a state of lower drag when compared to its undisturbed counterpart. Furthermore, recall that the particle position is the same for all these data and that the meniscus profiles do not depend on $\mu^{*}$. Therefore, for either $\alpha=75^{\circ}$ or $\alpha=110^{\circ}$, it is not too surprising to see that the shift of $F_{D}^{*}$ with respect to unity grows as the viscosity mismatch is further increased. Coming back to Fig. 6 , it means that both the curve asymmetry, with respect to $\alpha=90^{\circ}$, and the well depth, with respect to unity, would be enhanced for much lower values of $\mu^{*}$. This statement suggests that the larger the viscosity mismatch, the larger the drag 


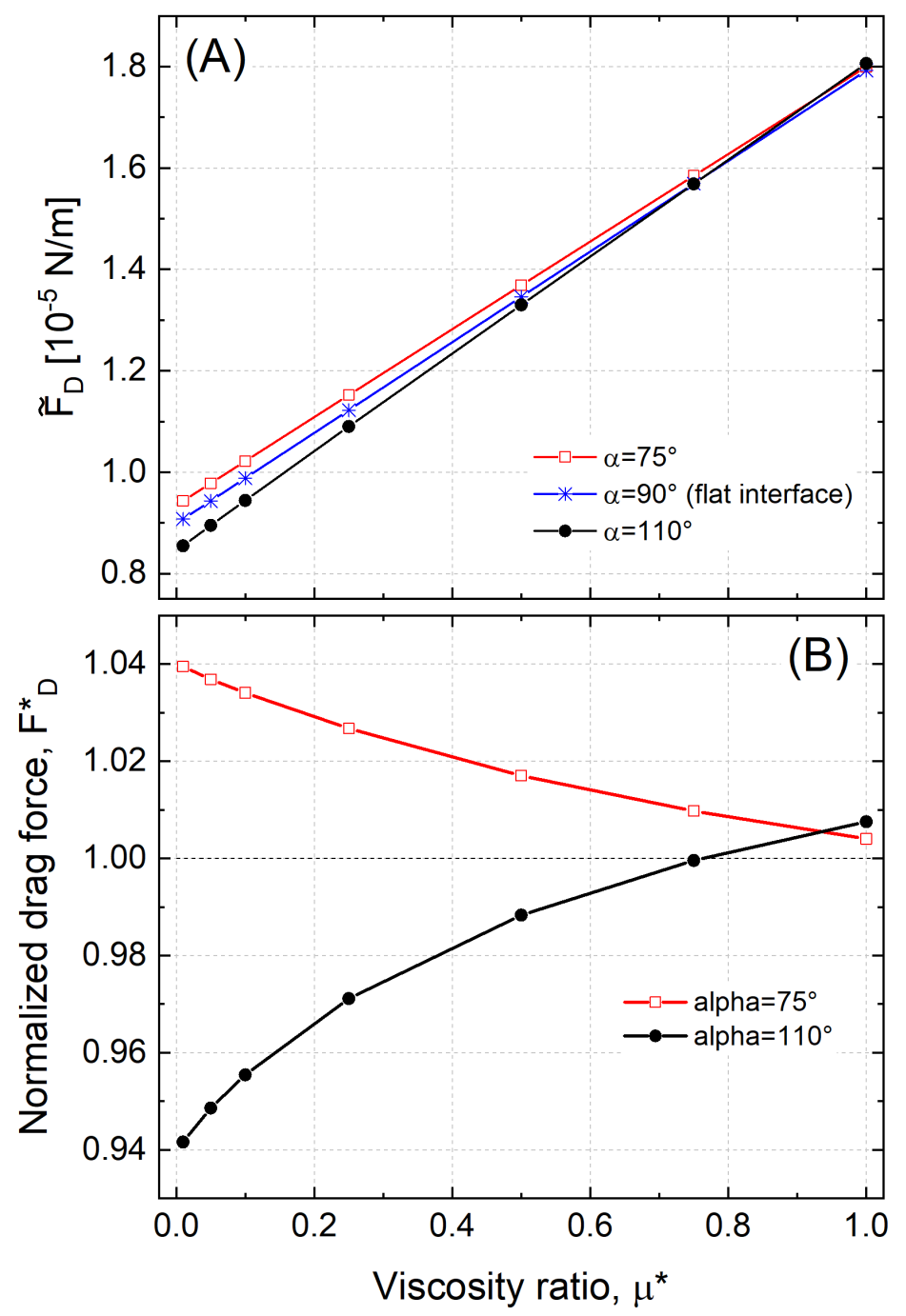

Figure 7: (A) Computed dimensional drag force $\left(\tilde{F}_{D}\right)$ as a function of the viscosity ratio $\left(\mu^{*}\right)$ for three different contact angles $(\alpha)$. (B) Corresponding normalized drag forces $\left(F_{D}^{*}\right)$ vs. $\mu^{*}$. The data for $\alpha=110^{\circ}$ clearly evidence that the presence of interfacial deformations can result in a configuration of lower drag when compared to an undisturbed fluid interface. Simulation parameters: $\rho^{*}=0.8, C n=0.04$. 
enhancement or reduction. For instance, the maximum excess drag force in Fig. 6 does increase from $\simeq 4.5 \%$ for $\mu^{*}=1$ to $\simeq 6 \%$ for $\mu^{*}=0.75$. The use of smaller viscosity ratios, such as $\mu^{*}=0.02$, actually makes the maximum excess drag go above $10 \%$ for $\alpha=45^{\circ}$ and $\rho^{*}=0.8$ (data not shown). An equally important drag reduction occurs for $\alpha=135^{\circ}$ in this case.

\subsection{Influence of the particle density}

In the preceding section, the particle's center of mass was located at the center of the simulation box and we examined the influence of various contact angles and viscosity ratios on the drag force exerted on the particle. In this section, we wish to investigate the effect of the particle density for a given contact angle. One way to simulate either a heavy or light particle is to displace its vertical position $\left(y_{p}\right)$ with respect to the center of the box in the $y$-direction, as explained in Secs. $3.3 \& 5.2$. We consider two contact angles, namely $\alpha=110^{\circ}$ and $\alpha=75^{\circ}$, and vary $y_{p}$ in the range $[-0.4 R, 0.4 R]$. In each case, we compute the drag force, the particle density, and the meniscus elevations. The resulting numerical data are graphed in Fig. 8.

We only consider the case $\mu^{*}=1$, which is the simplest situation to deal with for investigating the effect of the particle density. For each $\alpha$, we first notice that the curves $F_{D}^{*}$ vs. $y_{p}$ (Fig. 8a) are not symmetrical with respect to $y_{p}=0$. This is expected since for $\alpha \neq 90^{\circ}$, the meniscus computed for $y_{p}$ differs from that obtained for $-y_{p}$. Furthermore, the values of $F_{D}^{*}$ are all greater than 1 for both contact angles but again, the excess drag is rather moderate. It is slightly above $2.5 \%$ (resp., $2 \%$ ) at most for $\alpha=110^{\circ}$ (resp., $\alpha=75^{\circ}$ ) with our simulation parameters. The corresponding particle densities are plotted in Fig. 8b. They follow the expected trend, i.e. the heavier (resp. lighter) the particle, the more it sinks (resp. rises) into the lower (resp. upper) fluid.

The meniscus elevations exhibited on Fig. $8 \mathrm{c}$ deserve some comments. First of all, a striking feature emerges when comparing Figs. 8a,c: we note that the highest values of $F_{D}^{*}$ do not correlate with the largest meniscus elevations as in Fig. 4, and, rather surprisingly, it is actually even the opposite. See for instance the values of $F_{D}^{*}$ and $\left|\Delta y_{m}\right|$ obtained at 


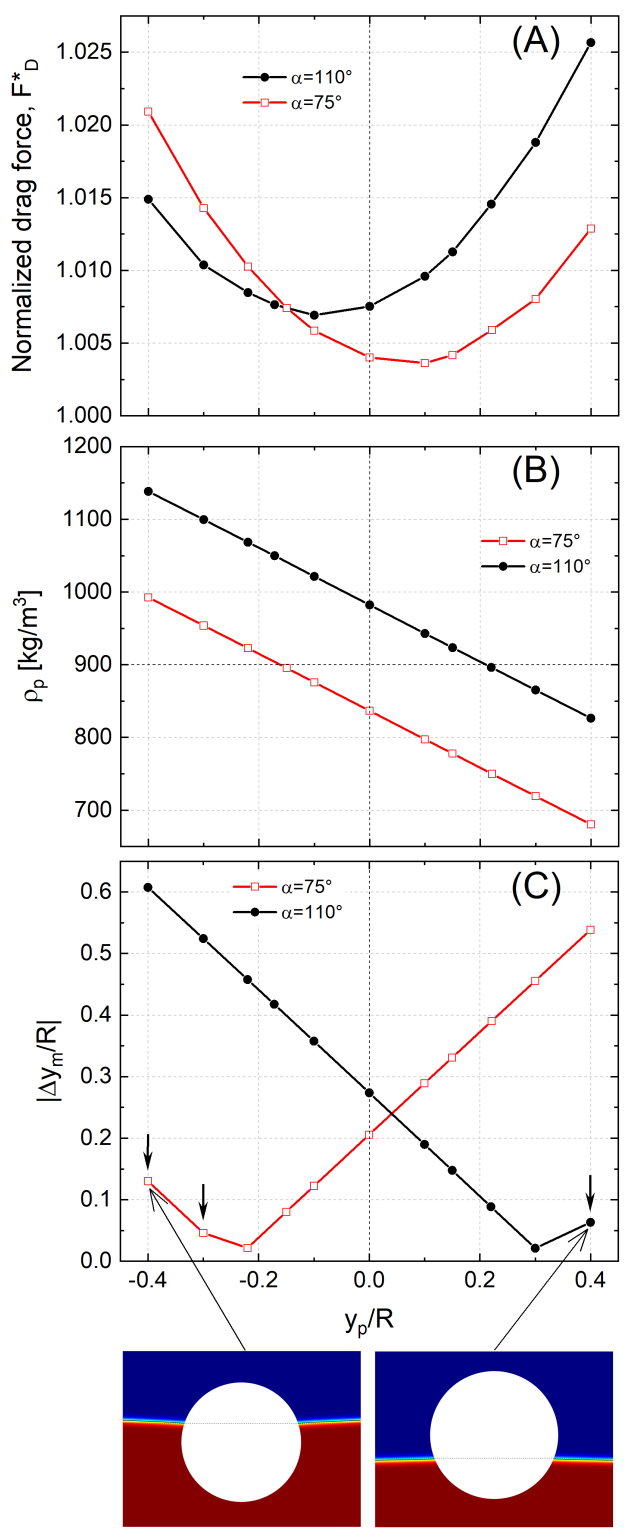

Figure 8: (A) Computed normalized drag force $\left(F_{D}^{*}\right)$ as a function of the particle position across the interface $\left(y_{p}\right)$ for two different contact angles $(\alpha)$. (B) Corresponding particle densities $\left(\rho_{p}\right)$ calculated from the interfacial profiles as explained in Secs. $3.3 \& 5$. Associated meniscus elevations $\left(\left|\Delta y_{m}\right|\right)$. The black arrows mark a change in the meniscus curvature, which is illustrated by the two zoomed-in phase-field snapshots at the bottom. Simulation parameters: $\mu^{*}=1, \rho^{*}=0.8, C n=0.04$. 
$y_{p}= \pm 0.3 R$ on Figs. 8a,c. Therefore, for a given contact angle, it seems like the amount of drag is primarily controlled by the particle position across the interface, and not only by the interfacial distortions. Otherwise stated, the same value of $\left|\Delta y_{m}\right|$ may well correspond to two different values of $F_{D}^{*}$ depending on $y_{p}$. And this is indeed well-illustrated for e.g. $\alpha=75^{\circ}$, where $\left|\Delta y_{m}\right|$ is about the same for both $y_{p}=-0.1 R$ and $y_{p}=-0.4 R$, but the corresponding drag forces differ significantly (Fig. 8a).

Next, we note that the minimum drag in Fig. $8 \mathrm{a}$ is obtained for small particle displacements $\left(y_{p} \simeq \pm 0.1 R\right)$ and moderate $\left|\Delta y_{m}\right|(<0.2 R)$. Further increasing $\left|y_{p}\right|$ always results in an enhanced drag force, even if $\left|\Delta y_{m}\right|$ drops substantially. One might argue that the increase in $F_{D}^{*}$ for large values of $\left|y_{p}\right|$ is the result of a confinement effect, i.e. one of the walls being a bit closer to the particle surface. However, using single-phase flow simulations (Sec. 5), we checked that confinement effects manifest themselves only when the particle surface is typically less than one diameter away from the moving wall. In all the configurations considered in Fig. $8, y_{p}$ ranges from $-0.4 R$ to $0.4 R$ and the separation distance between the cylinder surface and the walls always exceeds several diameters. Consequently, one may rule out any influence of the confining walls on the drag force values when the particle is moved across the fluid interface.

Finally, the solid arrows on Fig. 8c mark the cases where the meniscus curvature changes sign. More precisely, for those values of $y_{p}$, the otherwise concave (resp. convex) meniscus normally obtained for $\alpha=110^{\circ}$ (resp. $\alpha=75^{\circ}$ ) cannot be retained and it gradually becomes flat and then convex (resp. concave). The curvature reversal is illustrated by the two zoomed-in phase-field snapshots at the bottom of the graph. This 'meniscus transition' goes in pairs with a new increase in $\left|\Delta y_{m}\right|$ and is responsible for the non-monotonic variation of $\left|\Delta y_{m}\right|$ vs. $y_{p}$ (Fig. 8c).

\section{Concluding remarks}

The primary goal of the present study was to explore, through 2D numerical simulations, the influence of interfacial distortions on the drag force exerted on a particle straddling a fluid interface. We mainly focused on the effects of three parameters, the contact 
angle, the viscosity ratio, and the particle density. For fluids of equal viscosity and the particle centered on the initially flat interface, our results reveal that the drag force is an increasing function of interfacial deformations with the minimum drag obtained with a flat interface. Introducing a viscosity difference between the two fluids alters this intuitive picture as a state of lower drag may actually be achieved with a non-flat interface rather than with a flat one. Furthermore, high viscosity mismatches seem to favor either large drag enhancement or large drag reduction depending on the contact angle.

Other counter-intuitive findings are found when tuning the particle density. In this case, with matched fluid viscosities, we have shown that the drag force is again not necessarily an increasing function of interfacial deformations. Indeed, "high" drag configurations are possible even with small meniscus distortions depending on how the particle is positioned across the interface. This result has never been predicted nor experimentally evidenced before. Thus, based on the above findings, two main interesting insights emerge: (i) large drag forces may not always correspond to large interfacial distortions, and (ii), the drag force is strongly affected by the location of the particle across the interface.

Another salient result is that interfacial deformations do not seem to yield significant drag variations compared to the case of a flat interface. As an upper bound order of magnitude, our simulation data indicate that interfacial distortions may increase or decrease the drag coefficient by about $10 \%$ within the explored physical parameters. This result is the first simulated estimate of the influence of fluid interface perturbations on the mobility of interfacial particles. It is consistent with drag enhancement observed in experiments involving liquid-liquid interfaces [21, 22, 23] but larger differences exist with data compiled from experiments performed at liquid-gas interfaces [14]. However, in the latter case, the physical parameters greatly differ from those we used in our study. Nevertheless, let us recall here that a painstaking comparison between simulations and experiments was beyond the scope of the present work, whose primary objective was to capture qualitative trends through an approximate 2D approach.

Overall, our study unearthed unexpected findings on a rather simple situation where the interfacial deformations were caused by the buoyant weight of macroscopic particles. 
While this situation applies down to length scales, say, $\approx 50 \mu \mathrm{m}$, it is worth pointing out that interfacial distortions also exist when dealing with much smaller adsorbed particles. In this case, gravity is no longer relevant but the particle' surface roughness, the chemical inhomogeneities, the presence of surface electric charges or the nonspherical shape all serve as other potential sources of interfacial perturbations, possibly with undulating contact lines as well $[2,4,41]$. How do these phenomena impact drag forces? Such issues pertain to a much broader class of colloidal systems, including biological membranes, and definitely warrant more in-depth investigations. We hope that the present study will foster the development of other simulations or theoretical calculations on the topic we have touched upon herein.

\section{Acknowledgements}

This work was financially supported by the EU Marie-Curie fellowship 'CoPEC' under grant No 794837-H2020-MSCA-IF-2017 and by the NSERC Discovery grant No 201904162. One of us (J.-C.L.) is also indebted to the University of Bordeaux for further financial support thanks to the IdEx program entitled "Développement des carrières Volet personnel de recherche". M.Q. wishes to thank for the support of the four-year Doctoral Fellowship of the University of British Columbia. The IT staff of the Mathematics department of the University of British Columbia is also gratefully acknowledged for their valuable help and support.

\section{Author contributions}

J.-C.L. and J.J.F. designed research; J.-C.L. and M.Q. performed research; all authors analyzed the data, interpreted the results and collaborated on the manuscript.

\section{Appendix: Derivation of Eq. (23)}

The details for deriving Eq. (23) in Sec. 6.1 can be found in [38]. Here, we summarize 
the main steps of the calculations.

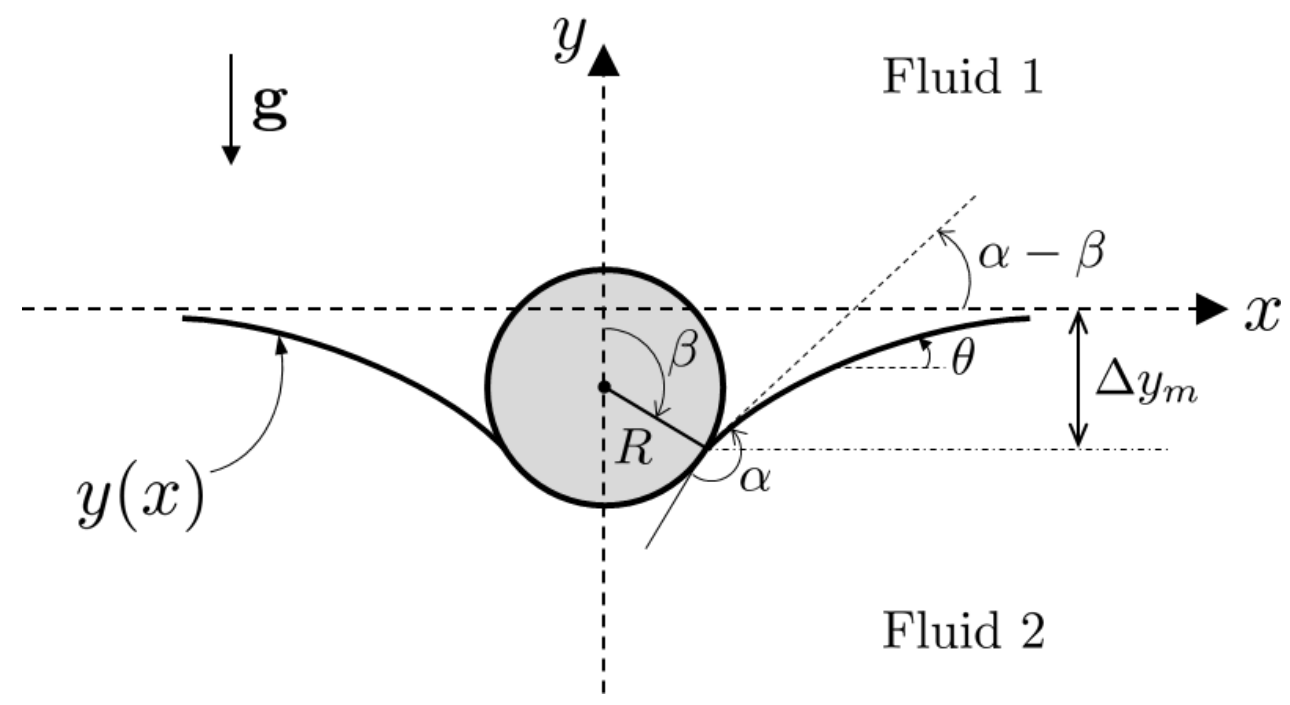

Figure 9: Geometry of the floating circular cylinder used to derive Eq. (23) in the appendix. The fluid interface is defined by the function $y(x)$. Definitions of symbols: $R$, cylinder radius; $\alpha$, contact angle; $\beta$, floating angle; $\theta$, interface slope; $\Delta y_{m}$, meniscus elevation.

With the notations of Fig. 9, we consider a static situation where a circular cylinder floats at a fluid interface, which is described by the function $y(x)$. We set the origin of the $y$-axis at the position of the flat interface far from the particle. Since the interface curvature, noted as $\kappa$, also tends to zero far from the particle, the hydrostatic YoungLaplace equation governing the shape of the $2 \mathrm{D}$ curved fluid interface in our problem is written as

$$
\kappa=-\frac{y}{L_{c}^{2}},
$$

where $L_{c}$ is the capillary length introduced in Sec. 3.2. The curvature is given by

$$
\kappa=-\frac{y^{\prime \prime}}{\left(1+y^{\prime 2}\right)^{3 / 2}}=\frac{1}{y^{\prime}}\left(\frac{1}{\sqrt{1+y^{\prime 2}}}\right)^{\prime}=\frac{1}{y^{\prime}} \frac{d|\cos \theta|}{d x},
$$

where $y^{\prime}=d y / d x=\tan \theta$, as shown in Fig. 9, with the prime denoting a derivative with respect to $x$. Combining Eq. (24) with the last two expressions of Eq. (25) and rearranging, we obtain the following nonlinear differential equation for the fluid interface:

$$
\frac{d}{d x}\left(\frac{1}{\sqrt{1+y^{\prime 2}}}\right)=\frac{d|\cos \theta|}{d x}=-\frac{y y^{\prime}}{L_{c}^{2}}=-\frac{\left(y^{2}\right)^{\prime}}{2 L_{c}^{2}} .
$$


Integrating once with respect to $x$, we get

$$
\frac{1}{\sqrt{1+y^{\prime 2}}}=|\cos \theta|=-\frac{y^{2}}{2 L_{c}^{2}}+C,
$$

where $C$ is a dimensionless integration constant. Requiring that both $y$ and $\theta$ decay to zero as $x$ tends to infinity, we obtain $C=1$. At the contact line, we have $\theta_{\mathrm{cl}}=\alpha-\beta$ and $y_{\mathrm{cl}}=\Delta y_{m}$ (see Fig. 9), so that the last two expressions of Eq. (26) give

$$
\frac{\left(\Delta y_{m}\right)^{2}}{2 L_{c}^{2}}=1-\cos (\alpha-\beta)=2 \sin ^{2}\left(\frac{\alpha-\beta}{2}\right),
$$

where the absolute value has been dropped since the angle $(\alpha-\beta)$ is always smaller than $\pi / 2$. Finally, Eq. (27) yields Eq. (23)

$$
\left|\Delta y_{m}\right|=2 L_{c} \sin \left(\frac{\alpha-\beta}{2}\right) \text {. }
$$

\section{References}

[1] Kralchevsky PA, Nagayama K (2000) Capillary interactions between particles bound to interfaces, liquid films and biomembranes. Adv. Coll. Interf. Sci. 85:145-192.

[2] Danov KD, Kralchevsky PA (2010) Capillary forces between particles at a liquid interface: General theoretical approach and interactions between capillary multipoles. Adv. Coll. Interf. Sci. 154:91-103.

[3] McGorty R, Fung J, Kaz D, Manoharan VN (2010) Colloidal self-assembly at an interface. Materials Today 13(6):34-42.

[4] Dasgupta S, Aust T, Gompper G (2017) Nano- and microparticles at fluid and biological interfaces. J. Phys.: Condens. Matter 29(37):373003 (41pp).

[5] Du K, Liddle JA, Berglund AJ (2012) Three-dimensional real-time tracking of nanoparticles at an oilwater interface Langmuir 28:9181-9188. 
[6] O’Neill ME, Ranger KB, Brenner H (1986) Slip at the surface of a translatingrotating sphere bisected by a free surface bounding a semiinfinite viscous fluid: Removal of the contact line singularity. Phys. Fluids 29(4):913-924, and references therein.

[7] Brenner H, Leal LG (1978) A micromechanical derivation of Fick's law for interfacial diffusion of surfactant molecules. J. Colloid Interface Sci. 65(2):191-209.

[8] Danov KD, Aust R, Durst F, Lange U (1995) Influence of the surface viscosity on the hydrodynamic resistance and surface diffusivity of a large Brownian particle. $J$. Colloid Interface Sci. 175:36-45.

[9] Danov KD, Dimova R, Pouligny B (2000) Viscous drag of a solid sphere straddling a spherical or flat interface. Physics of Fluids 12:2711-2722.

[10] Pozrikidis C (2007) Particle motion near and inside an interface. J. Fluid Mech. 575:333-357.

[11] Ally J, Amirfazli A (2010) Magnetophoretic measurement of the drag force on partially immersed microparticles at airliquid interfaces. Colloids and Surfaces A: Physicochem. Eng. Aspects 360:120-128.

[12] Dani A, Keiser G, Yeganeh M, Maldarelli C (2015) Hydrodynamics of particles at an oil-water interface. Langmuir 31:13290-13302.

[13] Dörr A, Hardt S, Masoud H, Stone HA (2016) Drag and diffusion coefficients of a spherical particle attached to a fluid interface. J. Fluid. Mech. 790:607-618.

[14] Petkov JT, Denkov ND, Velev OV, Danov KD, Aust R, Durst F (1995) Measurement of the drag coefficient of spherical particles attached to fluid interfaces. J. Colloid Interface Sci. 172:147-154.

[15] Petkov JT, Danov KD, Denkov ND, Aust R, Durst F (1996) Precise method for measuring the shear surface viscosity of surfactant monolayers. Langmuir 12:26502653. 
[16] Dimova R, Danov KD, Pouligny B, Ivanov IB (2000) Drag of a solid particle trapped in a thin film or at an interface: Influence of surface viscosity and elasticity. J. Colloid Interface Sci. 226:35-43.

[17] Fischer TM, Dhar P, Heinig P (2006) The viscous drag of spheres and filaments moving in membranes or monolayers. J. Fluid Mech. 558:451-475.

[18] Boniello G, Blanc C, Fedorenko D, Medfai M, Mbarek NB, In M, Gross M, Stocco A, Nobili M (2015) The viscous drag of spheres and filaments moving in membranes or monolayers. Nat. Mater. 14:908-913.

[19] Koplik J, Maldarelli C (2017) Diffusivity and hydrodynamic drag of nanoparticles at a vapor-liquid interface. Phys. Rev. Fluids 2:024303-1-12.

[20] Das S, Koplik J, Farinato R, Nagaraj DR, Maldarelli C, Somasundaran P (2018) The translational and rotational dynamics of a colloid moving along the air-liquid interface of a thin film. Scientific Reports 8:8910-1-13.

[21] Vassileva ND, van den Ende D, Mugele F, Mellema J (2005) Capillary forces between spherical particles floating at a liquid-liquid interface. Langmuir 21:11190-11200.

[22] Boneva MP, Christov NC, Danov KD, Kralchevsky PA (2007) Effect of electric-field induced capillary attraction on the motion of particles at an oil-water interface. Phys. Chem. Chem. Phys. 9:6371-6384.

[23] Boneva MP, Danov KD, Christov NC, Kralchevsky PA (2009) Attraction between particles at a liquid interface due to the interplay of gravity- and electric-field-induced interfacial deformations. Langmuir 25(16):9129-9139.

[24] Dörr A, Hardt S (2015) Driven particles at fluid interfaces acting as capillary dipoles. J. Fluid. Mech. 770:5-26.

[25] Wörner M (2012) Numerical modeling of multiphase flows in microfluidics and micro process engineering: a review of methods and applications. Microfluid Nanofluid $12: 841-886$. 
[26] Jacqmin D (1999) Calculation of two-phase NavierStokes flows using phase-field modeling. J. Comput. Phys. 155:96-127.

[27] Yue P, Feng JJ, Liu C, Shen J (2004) A diffuse-interface method for simulating two-phase flows of complex fluids. J. Fluid Mech. 515:293-317.

[28] Pigeonneau F, Hachem E, Saramito P (2019) Discontinuous Galerkin finite element method applied to the coupled unsteady Stokes/Cahn-Hilliard equations. Int. J. Numer. Meth. Fluids 90:267-295.

[29] Yue P, Zhou C, Feng JJ, Ollivier-Gooch CF, Hu HH (2006) Phase-field simulations of interfacial dynamics in viscoelastic fluids using finite elements with adaptive meshing. J. Comput. Phys. 219:47-67.

[30] Yue P, Zhou C, Feng JJ (2010) Sharp-interface limit of the Cahn-Hilliard model for moving contact lines. J. Fluid Mech. 645:279-294.

[31] COMSOL Multiphysics ${ }^{\circledR}$ version 5.4 reference manual. See https://www.comsol. com/documentation.

[32] Guyon E, Hulin JP, Petit L, Mitescu CD (2015) Physical Hydrodynamics (Oxford University Press 2nd Ed.).

[33] Khalili A, Liu, B (2017) Stokes' paradox: creeping flow past a two-dimensional cylinder in an infinite medium. J. Fluid Mech. 817:374-387.

[34] Faxén OH (1946) Forces exerted on a rigid cylinder in a viscous fluid between two parallel fixed planes. Proc. R. Swed. Acad. Eng. Sci. 187:1-13.

[35] Ben Richou A, Ambari A, Lebey M, Naciri JK (2005) Drag force on a circular cylinder midway between two parallel plates at $R e \ll 1$ Part 2: moving uniformly (numerical and experimental). Chem. Eng. Sci. 60:2535-2543.

[36] See chapter 18 of the COMSOL Multiphysics ${ }^{\circledR}$ reference manual [31] for details on deformed geometries and moving meshes. 
[37] The analytical expressions derived in [38] for the vertical force balance of a cylinder floating at a fluid interface are only valid in an unbounded geometry. Due to the finite size of the simulation box, we should therefore expect differences in the computed interfacial profiles when comparing the results. However, it turns out that these differences are very small, at most barely noticeable as mentioned in Sec. 5.2 (cf. Fig. 3). We believe that the box length, $L \simeq 6 L_{c}$ (cf. Table 1 ), is long enough so that confinement effects have a minimum impact on the meniscus shape.

[38] Pozrikidis C (2017) Fluid Dynamics. Theory, Computation, and Numerical Simulation (Springer 3rd Ed., New York). See chapter 5.

[39] Seppecher P (1996) Moving contact lines in the Cahn-Hilliard theory. Int. J. Engng. Sci. 34(9):977-992.

[40] Jacqmin D (2000) Contact-line dynamics of a diffuse fluid interface. J. Fluid Mech. 402:57-88.

[41] Botto L, Lewandowski EP, Cavallaro M Jr, Stebe KJ (2012) Capillary interactions between anisotropic particles. Soft Matter 8:9957-9971. 